\title{
Use Data Fragment Technology to Speed Queries of Mass Data
}

\author{
Ouyang Hao, Lu Xiaoyong, Yang Ping \\ Nanchang University \\ Nanchang, China \\ ouyanghao@ncu.edu.cn
}

\begin{abstract}
Based on the study about the common methods of speeding queries, a new project of using data fragment technology to speed queries of mass data has been advanced. At the same time, the technologies of database synchronization and load balance scheduling are also solved in the project. As an example, the solution of the public security management information system in hotels shows how to realize it.
\end{abstract}

Keywords- data fragment;database synchronous;distributed database;oracle; data replication

\section{MASS DATA RESTRICTS QUERIES OF INFORMATION}

With the development of information technology and network technology, enterprises pay more and more attention to information construction. However, after long time cumulation, data in information system has reached million level, which certainly would slow query speed. Slow queries become the weakness of information system.

In recent years, Hotel industry in China has developed rapidly. Under the new security situation, the Ministry of Public Security takes construction of public security management information system in hotels as important content of "Golden Shield Project", it is break with this, all kinds of special trade security management information system construction is promoted overall. According to unified requirements of Ministry of Public Security, the Public Security Bureau of Jiangxi Province implemented the public security management information system in hotels before 2008 Beijing Olympic Games, and established a comprehensive platform to collect and store data of hotel industry for implementing unified functions such as online fugitive alarming and information inquiry. The query speed of the system was ideal for a long time, while time grows, mass data slows quires, which directly affect work of the police.

\section{II . COMMON METHODS OF SPEEDING QUERIES}

Many experts and scholars study on how to speed queries and put forward some solutions, which could be summarized as following:

A.Improve hardware environment to increase hardware processing ability

The common way is to renew database service and to increase physical memory to improve CPU operation speed and $\mathrm{I} / \mathrm{O}$ speed. However, it has little effect to mass data.

B. Use index technology, expand the index numbe.
Carefully analyze the field that may be used in query process to create index for commonly used field. However, the main problem is that excessive index would affect speed of updating data. And to some fields (such as 'sex' which has only 'male' and 'female'), create index cannot improve the inquiry speed, because all the tables have to be scanned if we query information by this field.

C. Partition store table and index

Some database management system (like Oracle) has the function to partition store table and index, by which we can do range partition according to different value of key words to map different partitions to different disks. To a certain extent, it can reduce I/O conflict of disk and improve system performance. But usually hardware of database server has the function of $\mathrm{I} / \mathrm{O}$ parallel access by using RAID technology. So partition has little effect to speed queries.

\section{SQL optimization technique}

The difference between good quality SQL statement and bad is more than a hundredfold. The SQL optimization technique focus on how to be high quality SQL on improving usability and queries. But related theory about SQL optimization technique is mature enough. It is believed that SQL of information system is such an optimization that speeding queries by SQL optimization technique has been exhausted.

We have tested in the public security management information system in hotels of Jiangxi Province. The system has more than 3000 million data in some tables and the database physical capacity reaches GB level, the above ways are unable to achieve ideal effect and waste the replaced devices. So we need explore some new methods to improve queries.

After studying, we proposed using data fragment technology in distributed database system to speed queries of mass data. By testing again and again, the project is proved to be practicable.

\section{THE PROJECT DESIGN}

\section{A. data fragment can make full use of computational device in distributed environment}

According to some strategy, data fragment of distributed database system divides global relationship $\mathrm{R}$ into several sub-relations $\mathrm{R}_{\mathrm{i}}$ (referred to as fragments), then assigns these fragments to different physical sites in the network and different sites in the server is responsible for their own 
fragment so computational devices in the distributed environment can be fully taken advantage of and the site's capacity and resources are fully used to improve the effectiveness of data processing. At the same time, all of these are transparent to users.

Data fragment is mainly divided into horizontal fragment and vertical fragment. Horizontal fragment divides all tuples of global relations into several disjoint subsets and each subset is a fragment of the relationship. It mainly adopts selection operation $\sigma_{\mathrm{F}}(\mathrm{R})$ of relational algebra, where $\mathrm{F}$ is the fragment conditions. Vertical fragment divides attribute set of global relations into a number of subsets, and do projection operation $\pi_{\mathrm{i} 1 \ldots \mathrm{im}}(\mathrm{R})$ in the subsets, where $\mathrm{i}_{\mathrm{j}}(1 \leq \mathrm{j} \leq$ $\mathrm{m}, 1 \leq \mathrm{m} \leq \mathrm{n}, \mathrm{n}$ is the degree of relationship) is the attribute number and each projection is vertical fragment.

\section{B.Phasic characteristics of data in information system facing the management}

Information systems in an organization consist of operational layer, management layer, strategic layer. Among them, operational layer is for the staff of based organization who carry out daily business operations, production, basic data collection with TPS (Transaction Processing System) and its real time is strong; management layer summaries, focus and analysis business data with management information system (narrow MIS, Management Information System), then generates a series of different reports by Middle managers in organization. Management layer needs the data with characteristics of stage and mass data in the past over time, and its real time is not required enough; strategic layer determines the organization's goals and develops and achieves long-term strategy and direction through DSS (Decision Support System) by senior managers or senior managers in the organization. Also, it's random, general, predictive, unstructured and so on.

Information system with mass data generally belongs to management and strategic layer, while mass data is made by gathering TPS basic business data of various underlying organizations, and data query and statistics are done on data in a period. So, business processing time as a boundary can be considered to translate mass data into periodically fragment, then deploy the newest high usage fragment in the high performance server and the historical low usage fragment in the old sever. However, neither does it slow down the query, nor replace the hardware frequently.

\section{Detailed overview of the project}

Now, take the public security management information system in hotels as an example to introduce the detailed overview of the project. Detailed system architecture is shown in Figure 1:

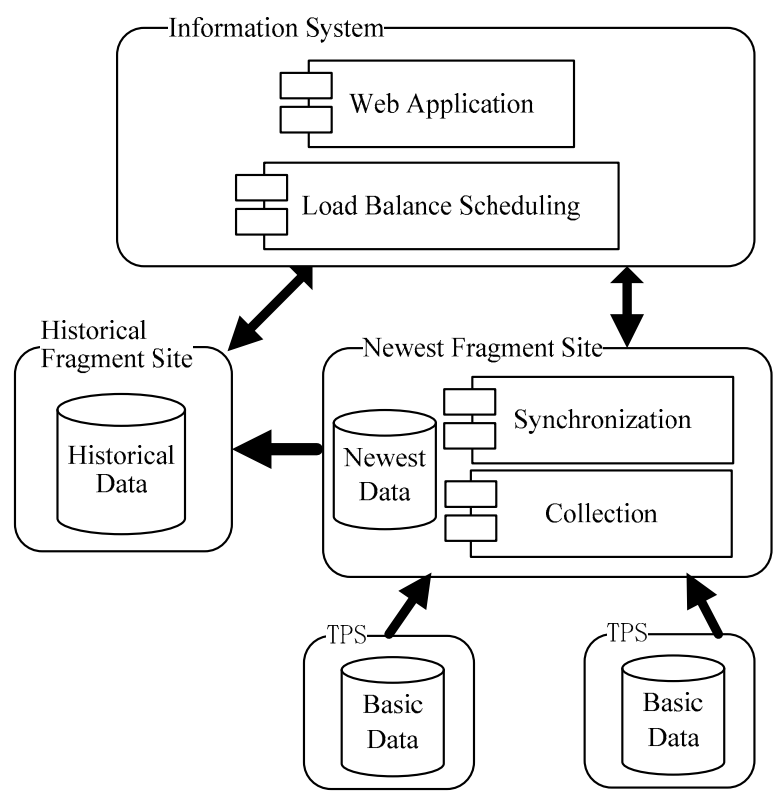

Figure 1. System architecture

Data horizontal fragment was adopted in the project and the select condition of fragment is business processing time. In consideration of data query of the project is mainly concentrated in the last two months, so we take data in the last two months as a fragment and deploy them in a new high performance server, fragment other historical data in years and deploy them in other servers.

The newest fragment site is in charge of collecting transactional data of the public security management information system in hotels in different regions. Data collection is one function of the system.

Because the newest fragment site only stores data in the last two months, Data synchronization must be implemented between the newest fragment site and historical site, so sync expired data to historical site and delete them from current site every day is needed.

The users use unified Web application interface to query data though data are fragmented and no concentrated in physics. So it requires professionals to add a load balance scheduling module in Web Application System ,which can judge time range of query data automatically and distribute SQL statement to relevant of DBMS for data processing.

\section{THE TECHNICAL PROBLEMS NEED TO BE SOLVED}

After dealing with the data fragment in centralized database, we need solve the problems of database synchronization and load balance scheduling.

\section{A. database synchronization}

There are two different kinds of synchronization strategy in distributed environment. They are complete synchronization and differences synchronization. Complete synchronization is to transmit all data in source database to target database, then update data by target database according to the data which is transmitted and synchronized 
logic. But its logic is simple that data transmission would be very large if there are many data in database. Differences synchronization, by contrast, only transmits changed data to target database, greatly saved data transmission and enhanced synchronization efficiency.

Data synchronization in this project is differences synchronization. The two databases are with the same construct and nature. Source database is the newest data fragment and target database as historical data fragment. Synchronization is in charge of syncing update data in newest data fragment to historical data fragment, in fact it's make a duplicate in historical data fragment from newest data fragment .

At present, large database management system provides data synchronization, basically have the following methods:

a) Trigger: Create trigger in table to track insert, update and delete operation for real-time executing data synchronization. While the biggest risk is transaction won't rollback until undo update transaction of source database, which would affect updating source data.

b) Advanced replication of data: Replication is advanced function of database system. It can copy data objects from based database to secondary database with different physical sites for supporting distributed application. When update data in based database, the changes will be automatically applied to secondary database. Replication can be divided into synchronous and asynchronous . Advanced replication of Oracle as an important part of Oracle, the user need do some configuration. Advanced replications of Oracle provide replication based on the whole table as well as partial table. The two schemes are implemented by two kinds of replication mechanism of Oracle, mainline replication and materialized view replication. To those who only need sync update part operation results (In the project, we need only sync Insert and Update), advanced replication can't implement it well.

c) Data snapshot: Snapshot of Oracle database is a table, which contains query result for one or more tables or views in local or remote database. That is hypostasis of snapshot is save query result in local or remote database in a table. There are two ways of refresh in snapshot: fast refresh and complete refresh. Fast refresh requires that there is snapshot $\log$ in main table of snapshot; When complete refresh, Oracle do snapshot query and save the result in snapshot. Because fast refresh need only transmit changed data via network from main database to snapshot, while complete refresh transmit all query result, fast refresh faster than complete refresh. The user can define refresh time of snapshot

d) Job technology: First define a procedure for implementing synchronization logic, and then define a database job. For executing synchronization logic, DBMS can call the procedure regularly or periodically. It's flexible for users can freely define synchronization function in procedure according to logic requires.

Data synchronization in the project combines snapshot and Job technology. Active of data synchronization is shown in Figure 2. Detailed realization procedure is as follows: a) Define a database link in source database and access target database via it. linkOldHotelDb is link name, police is one user of target database, the password is police123, dbserver is local Net server name created in source database server for connecting target database.

create database link linkOldHotelDb connect to police identified by police123 using 'dbserver';

b) Define snapshot log in source table which need be synced(table t ch pic).DBMS can automatically write data update $\log$ in source table to snapshot $\log$ table(mlog\$_t_ch_pic),mainly record primary key of source table(such as ID), snapshot time(field name: SNAPTIME\$\$), data updata operation type(field name: DMLTYPE\$\$, I means insert,D means delete ,U means update)and some other properties. create snapshot log on $t$ ch pic;

c) Define procedure for implementing synchronization logic in the project, we need only sync result of insert and update operation in source table.

create or replace procedure pr_sync_chpic as

cursor csInsPics is select a. * from t_ch_pic a where id in (select id from mlog\$_t_ch_pic where dmltype $\$ \$=^{\prime} I^{\prime}$ or dmltype $\left.\$=^{\prime} U^{\prime}\right)$;

begin

for this in csInsPics loop

insert into

(this.id,this.length,this.picture);

t_ch_pic@linkOldHotelDb values

--delete snapshot log record which has already synced to target database

delete mlog\$_t_ch_pic where id=this.id;

commit;

end loop;

delete mlog\$_t_ch_pic where dmltype $\$ \$=' D^{\prime}$; commit;

end;

d) create Job. call procedure(pr_sync_chpic) every 5 minutes for implementing synchronization of table(t_ch_pic) declare jobNo number;

begin

dbms_job.submit(jobNo,'pr_sync_chpic;',sysdate,'sysdate+5/(24*60)')

commit

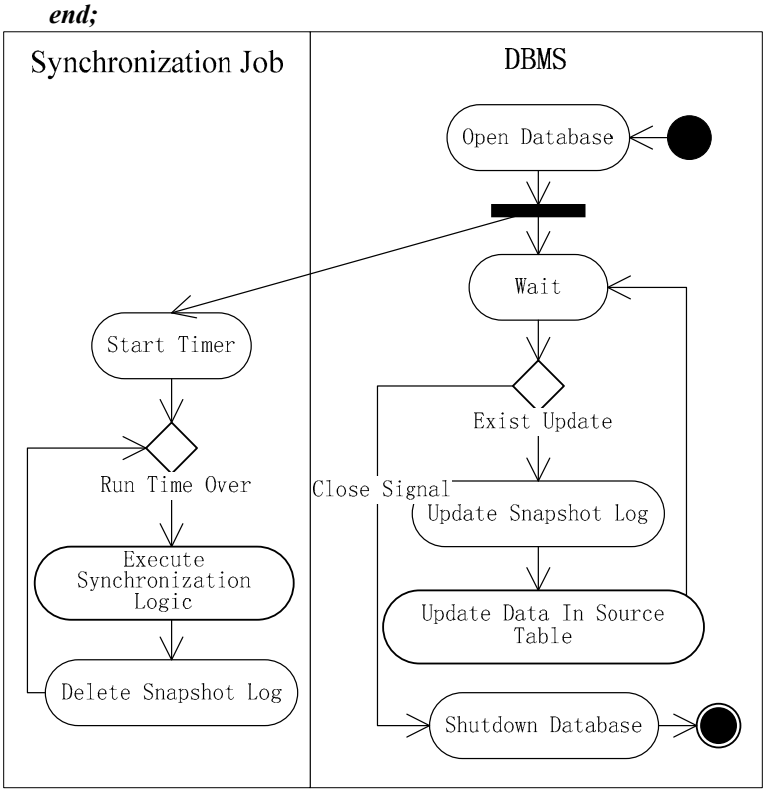

Figure 2. Active of data synchronization 


\section{B. Load Balance Scheduling}

Web application in centralized database information system is Browser/Web application server/Database server, and web application server connect with database server directly to access global database. After data distributed stored, database server become a whole which is composed of some machines. So, we should update business logic of Web application, add a load balance scheduling layer, to distribute global query business to distributed database server cluster. Then complete query by several machines and the results come together to result sets.

Suppose global database relation schema is $\mathrm{R}$, after distributed store, each fragments are $R_{1} 、 R_{2} 、 \ldots R_{n}$, in which $\mathrm{R}=\mathrm{R}_{1} \cup \mathrm{R}_{2} \cup \ldots \mathrm{R}_{\mathrm{n}}$. First, load balance scheduling layer analyze whether query business has specified business time range or not. If not, then query in global relation $\mathrm{R}\left(\mathrm{R}_{1} \cup \mathrm{R}_{2} \cup \ldots \mathrm{R}_{\mathrm{n}}\right)$ and complete query by all machine in cluster, or determine involved fragment of query according to the time range. But it may involve one fragment $\mathrm{Ri}$ or multi fragments ( involves one fragment, then distribute query business to machine which has $\mathrm{Ri}$, and finish query and return single result set $\mathrm{Si}$ by this machine. If many, divide query business into queries of different machines, and do operate by machine which has $\mathrm{Rk} 1$.then combine query results into a global result set $S=S_{1} \cup S_{2} \cup \ldots S_{m}$ and return result set $S$ to Web Application by the host machine.

\section{CONCLUSION}

Using data fragment technology to make every computational device joins the global calculation in centralized database can greatly speed queries of mass data in information system. The project got a satisfactory result for applying in the public security management information system in hotels, the query speed has been greatly improved.

\section{REFERENCES}

[1] TU Liming. A new technology of improve queries in Oracle $11 \mathrm{~g}[\mathrm{~J}]$,computer era, 2009(1),30 31

[2] DONG Jianquan, GUO Baokang, database principles turorial[M].BeiJing,Isinghua University Press,2007,203 205

[3] LIU Zhongying. Management information System[M].BeiJing:Higher Education Press, 2006

[4] Hassan A.Afyouni. Oracle9i Performance Tuning[M].BeiJing: Isinghua University Press, 2005,475 481

[5] LIN Yuan,CHEN Zhibo,Research and Apply of distributed heterogeneous database Sync system[J],Computer Engineering and Design, 2010 (24):78 81 